\title{
STATE OF THE ART IN DESIGN AND TECHNOLOGY OF SAW DEVICES
}

\author{
P. E. LAGASSE \\ Laboratory for Electromagnetism and Acoustics, University of Ghent, Ghent, Belgium
}

(Received May 5, 1979)

\begin{abstract}
In this paper the different classes of surface acoustic wave devices are briefly reviewed and examples of the state of the art are given. The fabrication techniques and the application of SAW components are also discussed.
\end{abstract}

\section{INTRODUCTION}

Elastic surface waves were first theoretically predicted by Lord Rayleigh ${ }^{1}$ at the end of the last century and they have been studied by geophysicists since then. It is only about 15 years ago that electronic engineers became interested in the application of Surface Acoustic Waves (SAW) to electronic signal processing devices. During those 15 years SAW technology has experienced a tremendous growth. As will be discussed in this paper, this can be attributed to a number of unique features of SAW devices, but the single most important factor has probably been the planar character of SAW technology. In this age of microelectronics, this compatibility with planar integrated circuit fabrication methods, has made possible the very rapid advances in the performances of SAW devices. It is the aim of this paper to give an overview of the state of the art in the various parts of the SAW technology. First the basic principles of SAW propagation and transduction will be briefly reviewed. Next transversal filters, perhaps the best known SAW components, will be discussed. The coded time domain devices, widely used in communications equipment and electronic warfare, are described in the following section. One of the latest and most promising developments in SAW components, namely the resonators, are analysed in Section 5, while the fabrication techniques for SAW devices are discussed in the last part of this paper.

\section{PROPERTIES AND TRANSDUCTION OF PIEZOELECTRIC SURFACE WAVES}

A Rayleigh surface wave propagates along the free surface of an elastic solid. This wave is dispersion free and guided by the surface of the elastic half space so that most of the wave energy is confined within a depth of one wavelength. On piezoelectric crystals this surface wave is accompanied by an electric field extending out of the substrate. This means that the piezoelectric surface wave can be tapped all along its propagation path, by means of metal electrodes deposited on the crystal surface. The basic component of all SAW devices is the interdigital transducer (IDT) shown in Figure 1. ${ }^{2}$

When the electric field, generated by the voltage applied between the fingers of the IDT, has a periodicity equal to the wavelength of the surface wave, a strong excitation of this wave takes place. The IDT in fact acts as an end-fire array. The electrical impedance of such an IDT is, to a good approximation, given by the equivalent network shown in Figure 2, where $C_{T}$ is the static capacitance of the IDT while the radiation resistance $R_{a}$ and reactance $X_{a}$ are given by:

$$
\begin{aligned}
& R_{a o}=2.87 \frac{1}{W \cdot \omega \cdot\left(\epsilon_{p}+\epsilon_{o}\right)} \cdot \frac{\Delta V}{V} \\
& R_{a}=R_{a o}\left(\frac{\sin F}{F}\right)^{2} ; X_{a}=R_{a o}\left(\frac{\sin 2 F-2 F}{2 F^{2}}\right) \\
& F=\pi N \frac{f-f_{o}}{f}
\end{aligned}
$$

where $N$ : the number of fingerpairs

$W$ : width of the transducer

$\Delta V$ : piezoelectric coupling coefficient for

$\bar{V}$ surface waves

$f_{o}$ : center frequency

One can see from Eq. (1) that it is possible to design the IDT in such a way as to obtain a given 

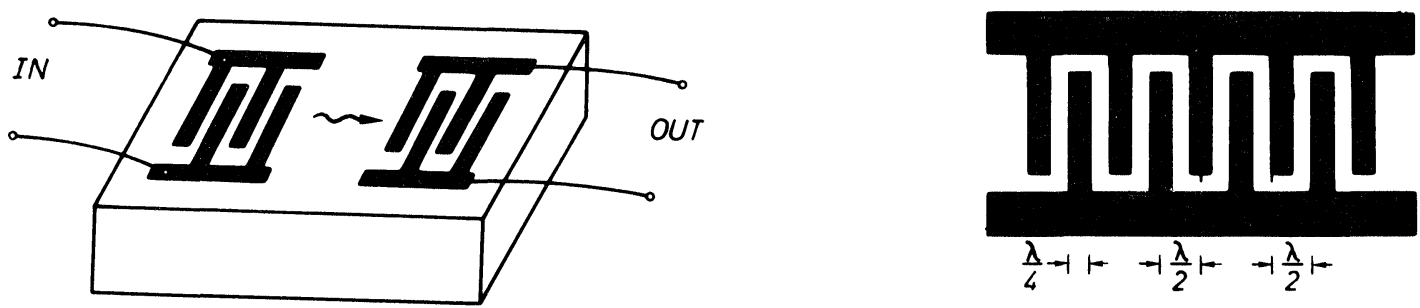

FIGURE 1 Interdigital transducers.

electrical impedance. Assuming lossless propagation the minimum insertion loss of a SAW delay line (Figure 1) is $6 \mathrm{~dB}$, due to the bidirectionality of the IDT.

Complicated transducer structures, containing many fingerpairs, cannot be analysed by means of the simple Eq. (1). In order to account for second order effects, such as acoustic reflections by the fingeredges, one has to use equivalent circuit models. ${ }^{3}$ In this method each finger is replaced by an equivalent circuit, so that the complete transducer is modelled by a complicated network of transmission lines and lumped circuit elements. Some of the more recent circuit models ${ }^{4,5}$ are quite sophisticated and are able to predict the response of any IDT very accurately over a broad frequency range. Such computer programs are necessary for analysing the variety of electrode structures that has evolved from the basic IDT. In order to avoid unwanteủ second order effects or to achieve special characteristics, various different electrode configurations have been proposed. Some of the more interesting are the split finger electrodes ${ }^{6}$ used to avoid reflections (Figure 3), and the three phase IDT $^{7}$ that allows undirectional operation.

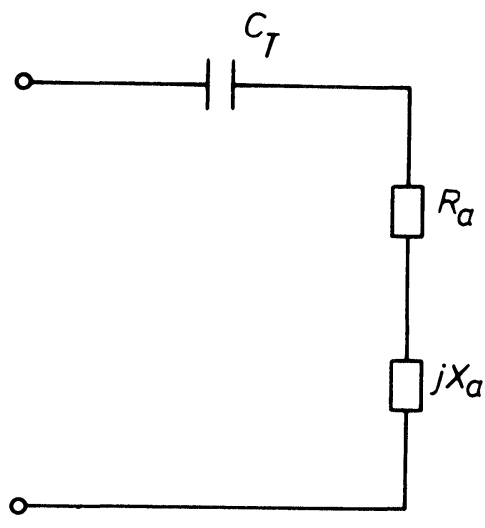

FIGURE 2 Equivalent circuit.

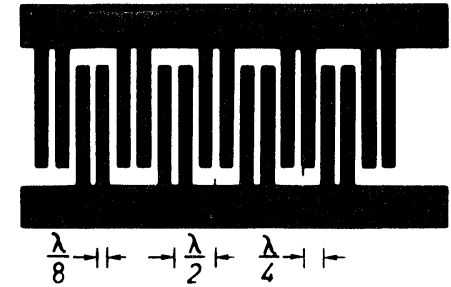

FIGURE 3 Normal IDT and double electrode IDT.

\section{SAW TRANSVERSAL FILTERS}

A transversal filter consists of a delay line containing taps at regular intervals $T$. Those taps are summed through a set of weighting networks $a_{n}$ to form the output of the transversal filter (Figure 4). If $f(t)$ is the electrical input signal, the output signal $g(t)$ can be written as:

$$
g(t)=\sum_{n=1}^{N} a_{n} f(n T)
$$

From this equation it follows that the transfer function of a transversal filter is the discrete Fourier transform of the tap weights $a_{n}$. Although a transversal filter can be implemented in several technologies, such as digital circuits or charge coupled devices, the implementation using surface acoustic waves has several advantages such as high frequency

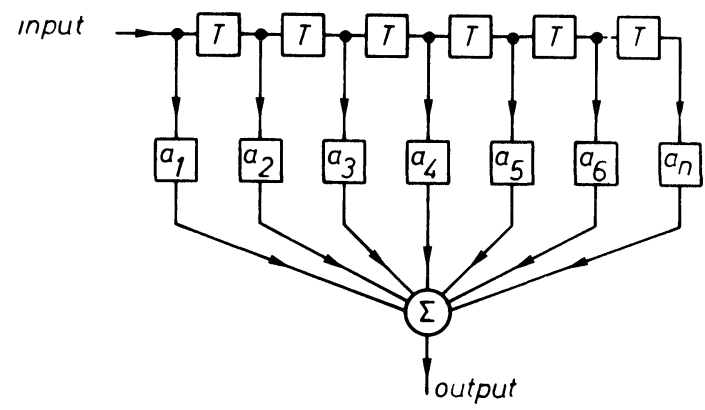

FIGURE 4 Transversal filter. 


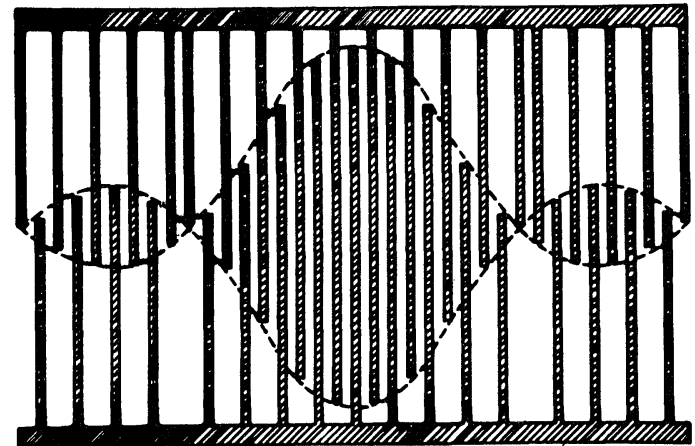

FIGURE 5 Apodised filter.

operation, simplicity of fabrication, and reliability due to the fact that it is a passive structure. In SAW transversal filters, weighted taps are usually realised by finger pairs with varying finger overlaps $a_{n}$

(Figure 5). Other tap weighting techniques have been proposed, such as finger-withdrawal weighting ${ }^{8}$ or capacitive weighting. ${ }^{9}$

For a specified filter characteristic, the required tap weights can readily be determined by means of the synthesis procedures used for digital finite impulse response filters. ${ }^{10}$ In order to obtain the desired frequency response, the computed finger overlaps should be corrected for a number of second order effects. Reflections of the surface wave on the edges of the fingers can be avoided by the use of split fingers. A penalty is the increase in resolution of the photolithography required to produce fingers with half the width of a normal finger. Depending on the crystal and its orientation, an IDT always radiates to some extent bulk waves. This can be particularly troublesome for achieving very low sidelobe stopbands in filters. One effective solution consists in the use of a "multistrip coupler". 11,12 As shown in Figure 6 this device allows one to transfer surface wave energy from one track to another, while the bulk waves continue straight through and are
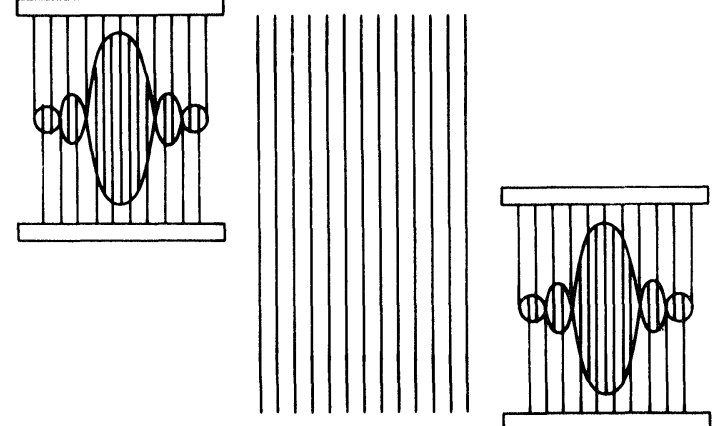

FIGURE 6 Transversal filter with multistrip coupler.

therefore not picked up by the output transducer. Although the multistrip coupler increases the size of the filter somewhat, it is widely used in SAW transversal filter design, because it also allows the two transducers to be independently apodised. If the distance between the transducers becomes large and if the finger overlap is comparable to the wavelength, diffraction loss has to be taken into account and the overlapping length must be corrected accordingly. ${ }^{13}$

Today the design of SAW transversal filters can take care of most second order effects and has reached such a level of perfection that filters can be synthesized with specifications on frequency response and group delay that could probably not be met by any other type of filter. An example of such filter is shown in Figure $7 .^{23}$

\section{CODED TIME DOMAIN DEVICES}

The advantages of SAW components for coded time domain devices can best be understood when one considers the very low velocity of surface waves. A typical velocity of $3300 \mathrm{~m} / \mathrm{sec}$ means that a $5 \mathrm{~cm}$ distance on the crystal contains a surface wave representing a $15 \mu \mathrm{sec}$ long part of the electrical
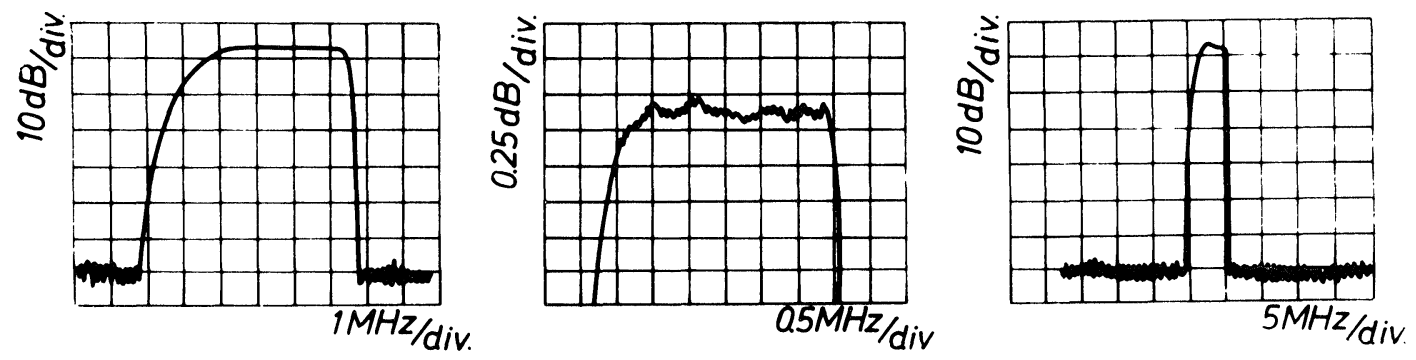

FIGURE 7 High performance SAW transversal filter (after Drummond and Roth). 

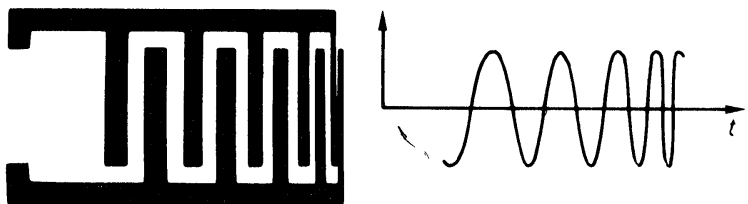

FIGURE 8 Impulse response of dispersive IDT.

signal. Through various tapping electrodes, this length of signal is available for various signal processing applications. Considering the fact that this signal is processed in real time and can contain frequencies up to $1 \mathrm{GHz}$, one can easily understand that such performances cannot be met by competing technologies such as CCD's or digital circuits.

Most coded time domain devices are matched filters. If $f(t)$ is the signal that has to be detected, the impulse response $g(t)$ of the matched filter is given by

$$
g(t)=f\left(t_{0}-t\right) .
$$

Since the electrode position and overlap forms, to a good approximation, the spatial replica of the impulse response of the IDT, it is relatively easy to synthesize matched filters using SAW devices (Figure 8). The two best known examples of SAW matched filters are the phase coded filters and the pulse compression filters. SAW biphase coded filters nowadays are realised with a number of chips up to 400 and with performances close to the theoretical maximum. An interesting survey of SAW phase coded filters is given by Collins et al. ${ }^{14}$ The pulse compression filter for chirp radar has been, and probably still is the foremost system application for SAW components. Time-bandwidth products of the order of 1000 can be realised by means of dispersive interdigital transducers. ${ }^{15}$ Even higher time-bandwidth products, up to 5000, can be obtained with reflective array compressors. ${ }^{16}$ As shown in Figure 9 the dispersive elements consist of two arrays of grooves, reflecting the surface wave to the output transducer. More recently, devices have been proposed where the grooves are replaced by reflecting dots. ${ }^{17}$

\section{SAW RESONATORS}

Quartz crystal resonators, using acoustic bulk waves, are very well known components in the electronic industry. Those resonators are based on the total reflection of bulk waves on the two parallel surfaces of the crystal and are limited for the fundamental mode of operation to frequencies below $50 \mathrm{MHz}$. In order to make SAW resonators it is necessary to have perfect reflectors for surface waves. It is not possible to reflect completely a surface wave on a sharp discontinuity since in that case, mode conversion into bulk waves will always take place. A solution to that problem lies in the use of distributed reflectors. A grating consisting of small surface discontinuities such as metal strips or grooves and with a periodicity of half a wavelength acts as a perfect mirror for surface waves without any mode conversion into bulk waves. ${ }^{18}$ A resonating cavity for surface waves is therefore formed by depositing two distributed reflectors, spaced by the right distance, on the surface of the crystal. ${ }^{19}$ Coupling with this SAW resonator is achieved by putting an IDT in the cavity spacing (Figure 10a). The impedance characteristic of the classic equivalent circuit (Figure 10b) for a crystal resonator, is also a very good approximation for the impedance of the SAW resonator. Quality factors of several 10000, with fundamental resonant frequencies to $1 \mathrm{GHz}$ or more have been obtained with SAW resonators.

Using distributed reflectors, interdigital transducers and multistrip couplers as basic building blocks, one can design a variety of single port and
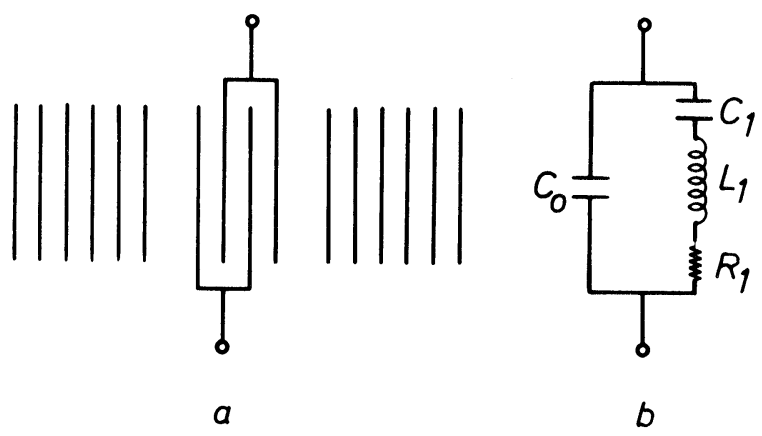

FIGURE 10 SAW resonator and equivalent circuits.

FIGURE 9 Reflective array compressor. 
multi port devices. It is beyond the scope of this paper to describe all the various configurations and their characteristics. An excellent review on this subject is given by Coldren and Rosenberg. ${ }^{20}$

\section{FABRICATION TECHNIQUES FOR SAW DEVICES}

SAW devices are basically fabricated by means of planar micro-electronic technology. The special requirements posed by SAW technology can be derived from the description of the devices given in preceding sections. The basic linewidth is equal to $\lambda / 4$ or $\lambda / 8$, where $\lambda$ is the acoustic wavelength. This means that, with a typical wave velocity of 3200 $\mathrm{m} / \mathrm{sec}$, the width of an electrode finger is equal to $6 \mu \mathrm{m}$ at $100 \mathrm{MHz}$ or to $0.75 \mu \mathrm{m}$ at $800 \mathrm{MHz}$. The total number of fingers in a SAW component can be of the order of several thousand. This combination of small linewidth with very high complexity patterns puts severe requirements on the mask fabrication and photolithography. Techniques such as projection printing or conformable mask shadow printing are normally used. In order to obtain the necessary precise control over the mark-space ratio of the IDT's the lift-off technique is often used. ${ }^{21}$ For very complex structures, such as reflective array compressors or resonator filters, electron beam lithography is needed. The grooves necessary for the fabrication of distributed reflectors for SAW resonators are made by ion bombardment etching. If no ion bombardment system is available one can also use a RF sputter etching system although this has some disadvantages, such as a less predictable etching rate due to the redeposition on the substrate of the removed material. Since a SAW device normally consists of one metal electrode structure with in some cases groove patterns, the number of fabrication steps is small. Very good reviews of fabrication techniques for SAW components are given by $\mathrm{Smith}^{21}$ and Matthews. ${ }^{2}$

\section{CONCLUSIONS}

From the very brief overview of SAW devices, given in the preceding sections, it appears that SAW technology has established itself as a viable component for a variety of signal processing systems. TV IF filters are well under way to become the first mass application for transversal SAW filters. SAW pulse compression filters have become important components of modern chirp radar filters. Phase coded SAW devices are more and more used in spread spectrum equipment. Oscillators in the VHF or UHF frequency band and based on either SAW delay lines or SAW resonators show excellent performance. One can therefore expect that the application of SAW devices will continue to grow in the coming years.

\section{REFERENCES}

1. Lord Rayleigh, On waves propagated along the plane surface of an elastic solid, Proc. London Math. Soc. 17 4-11 (1885).

2. G. A. Coquin and H. F. Tiersten, Analysis of the excitation and detection of piezoelectric surface waves in quartz by means of surface electrodes, J. Acoust. Soc. Amer., 41, 921-939 (1967)

3. W. R. Smith, H. M. Gerard, J. H. Collins, T. M. Reeder and H. J. Shaw, Analysis of interdigital surface wave transducers by use of an equivalent circuit model, IEEE Trans. Microwave Theory and Techn., MTT-17, 856-864 (1969)

4. R. Krimholtz, Equivalent circuits for transducers having arbitrary asymmetrical piezoelectric excitation, IEEE Trans $S U, 19427-436$ (1972)

5. W. R. Smith and W. F. Pedler, Fundamental- and harmonic frequency circuit-model analysis of interdigital transducers with arbitrary metallisation ratios and polarity sequences, IEEE Trans $M T T, 23,853-864$ (1975).

6. T. W. Bristol, W. R. Jones, P. B. Snow and W. R. Smith, Applications of double electrodes in acoustic surface wave design, IEEE Ultrasonics Symp. Proc., 343-345 (1972).

7. C. S. Hartmann, W. S. Jones and H. Vollers, Wideband unidirectional interdigital surface wave transducers, IEEE Trans. Sonics Ultrason, SU-19, 378-381 (1972).

8. C. H. Hartmann, Weighting interdigital surface wave transducers by selective withdrawal of electrodes, IEEE Ultrasonics Symp. Proc., 423-426 (1973).

9. D. C. Malocha and B. J. Hưnsinger, Capacitive tap weighted SAW transducers, IEEE Ultrasonics Symp. Proc., 411-413 (1975)

10. J. H. McClellan and T. W. Parks, A unified approach to the design of optimum FIR linear phase digital filters, IEEE Trans., CT-20 697 (1973).

11. F. G. Marshall, C. O. Newton and E. G. S. Paige, Theory and design of the surface acoustic wave multistrip coupler, Microwave Theory and Techn., MTT-21 206 (1973).

12. F. G. Marshall, C. O. Newton and E. G. S. Paige, Surface acoustic wave multistrip components and their applications, Microwave Theory and Techn., MTT-21 216 (1973)

13. T. L. Szabo and A. J. Slobodnik, Jr., Diffraction compensation in periodic apodized acoustic surface wave filters, IEEE Trans., SU-21 114 (1974)

14. J. H. Collins, P. M. Grant and B. J. Darby, Application of surface acoustic wave devices to spread spectrum communications, Wave Electronics, 1311 , (1976).

15. H. M. Gerard et al., Design and applications of highly dispersive acoustic surface wave filters, IEEE Trans., MTT-21 176 (1973).

16. R. C. Williamson, V. S. Dolat and H. I. Smith, L-band 
reflective array compressor with a compression ratio of 5120, IEEE Ultrasonics Symp. Proc., 490-492 (1973).

17. L. P. Solie, Reflective dot array devices, Ultrasonics Symposium Proceedings 579 (1978)

18. E. A. Ash, Surface wave grating reflectors and resonators, IEEE Symp. on Microwave Theory and Techn. (1970).

19. E. J. Staples, UHF surface acoustic wave resonators, Proc. 28th Frequency Control Symp., 280-285 (1974).

20. L. A. Coldren and R. L. Rosenberg, SAW resonator filter overview: design and performance tradeoffs, IEEE Ultrasonics Symp. Proc., 422 (1978).
21. H. I. Smith, Fabrication techniques for surface-acousticwave and thin-film optical devices, Proc. IEEE, 62, 1361 (1974).

22. H. Matthews, Surface Wave Filters Wiley, New York, (1977).

23. W. S. Drummond and S. A. Roth, Application of high performance SAW transversal filters in a precision measurement instrument, Ultrasonics Symp. Proc., 494 (1978). 

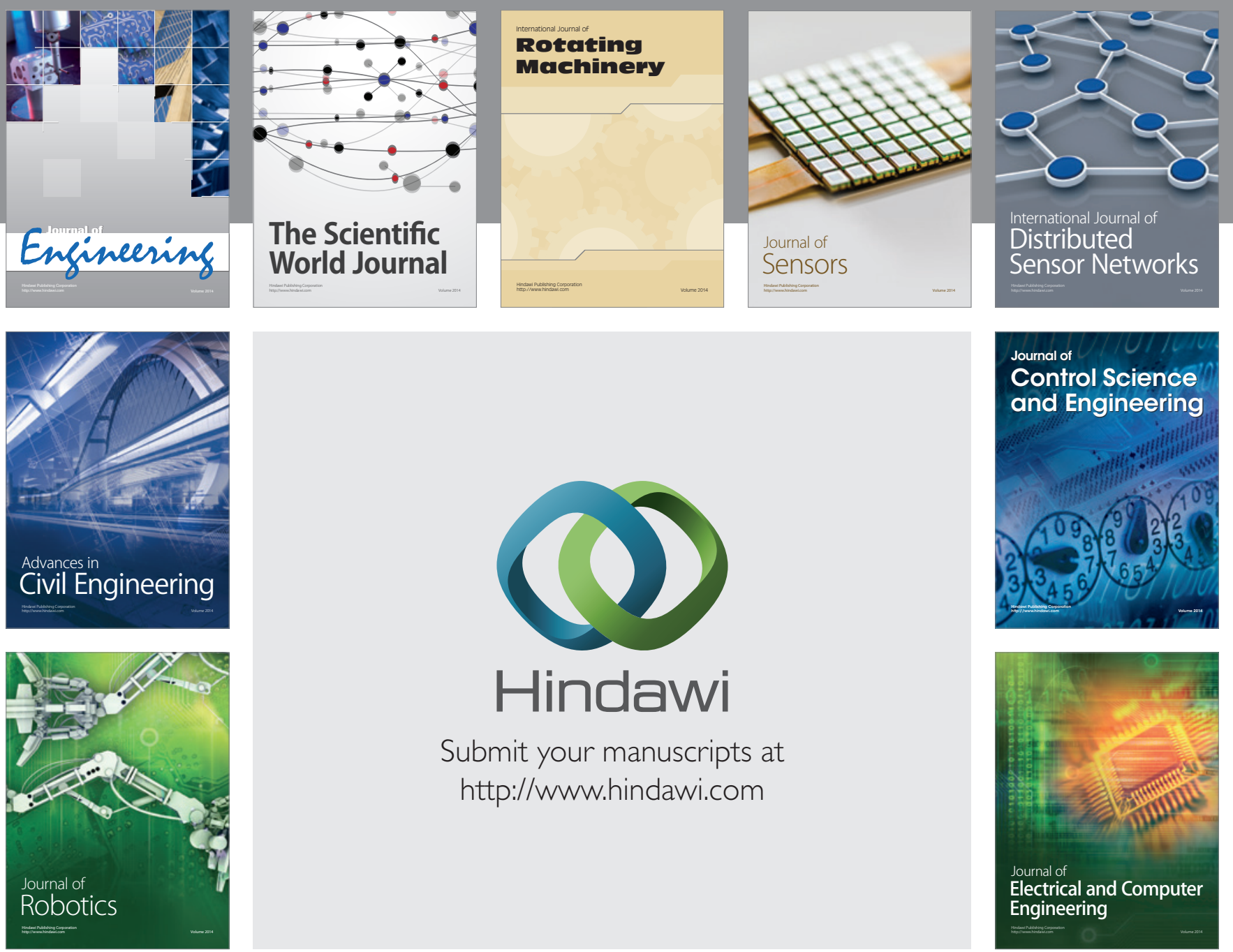

Submit your manuscripts at

http://www.hindawi.com
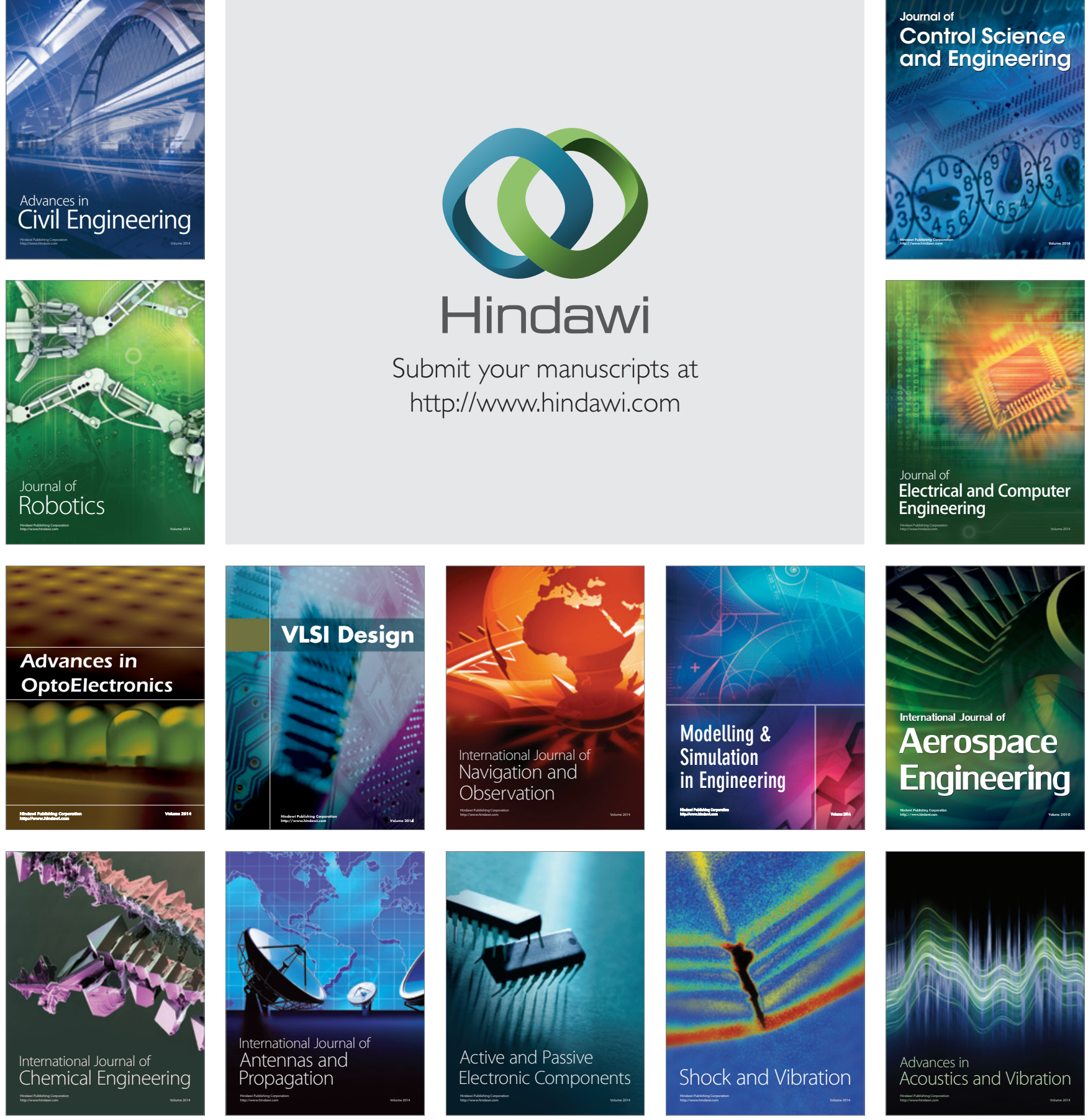